\title{
Modelling of the Parametric Behaviour of a Self-Heated Copper Vapour Laser: Issues Governing the Thermal Runaway Behaviour of the Plasma Tube Wall Temperature
}

\author{
$\underline{\text { R.J. Carman }}$
}

Centre for Lasers and Applications, Macquarie University, North Ryde, Sydney, NSW 2109, Australia

\begin{abstract}
A computer model has been used to simulate the discharge kinetics and parametric behaviour of a selfheated copper vapour laser for a wide range of optimum and non-optimum conditions. The results indicate that the ground state copper density and the peak electron temperature are the two most important parameters that affect laser performance. The results also confirm the existence of a threshold wall temperature (or threshold copper density) above which the plasma tube becomes thermally unstable with respect to the deposited electrical power, the wall temperature and the copper density, ultimately leading to thermal runaway. At low pulse repetition frequency (prf $<8 \mathrm{kHz}$ ), the thermal instability restricts the copper density (and laser output power) to values well below optimum.
\end{abstract}

\section{INTRODUCTION}

In the class of high average-power pulsed metal-vapour lasers (eg. Sr-He, $430.5 \mathrm{~nm} \& 416.2 \mathrm{~nm}$; Ba-Ne, $1.5 \mu \mathrm{m}, 2.55 \mu \mathrm{m}$; Ca-He, $370.6 \mathrm{~nm} \& 373.7 \mathrm{~nm})$, the copper laser $(\mathrm{Cu}-\mathrm{Ne}, 510.6 \mathrm{~nm} \& 578.2 \mathrm{~nm})$ is probably the most successful and universally recognised source of high-power radiation (typically up to 100W) at $\mathrm{kHz}$ repetition frequencies and with good wallplug efficiencies ( 1-2\%). Topical applications of copper vapour lasers (CVLs) include pumping solid-state media for frequency conversion to the ultraviolet[1] and infrared[2], laser guidestars[3], and industrial materials processing[4][5]. As part of the ongoing development of these devices, numerical modelling has been used to unravel the complex plasma kinetics of the pulsed glow discharge in the $\mathrm{Cu}-\mathrm{Ne}$ mixture comprising the laser active medium[6][7]. However, modelling studies of the parametric behaviour of CVLs, including analysis on both optimum and nonoptimum operating conditions, remain scarce in the literature[8]. This is undoubtedly largely due to the substantial computing time required to perform detailed calculations for a wide-range of operating conditions. However, the results from such a study could be used to clarify a number general issues concerning the plasma kinetics and the observed behaviour of CVLs, particularly relating to processes that ultimately limit the laser output power. The aim of the present study is to use an existing computer model[9] to generate a body of results describing the parametric behaviour of a single CVL device, and to compare these results with experimental data[10][11]. The results from such a study indicate that the laser output power is limited by thermal instability of the plasma tube wall temperature, particularly at low pulse repetition frequencies. This instability is caused by positive feedback between the electrical power deposition, the wall temperature, and the copper vapour density.

\section{THE MODEL}

The computer model used to generate the results in this paper has been described in detail elsewhere [9]. It is based on a spatially and time dependent rate-equation analysis of the plasma kinetics, including the external circuit, integrated over many excitation/afterglow cycles to yield temporally self-consistent results. A total of 15 atomic and ionic species of copper and neon are considered in addition to two intracavity laser flux intensities, a gas temperature, and around 70 electron collisional, heavy-body and radiative processes. A two-temperature (bi-Maxwellian) electron energy distribution function is used throughout to calculate the electron collision rates and plasma conductivity. The equations of continuity, energy conservation and momentum conservation are solved simultaneously for the electrons, ions and 


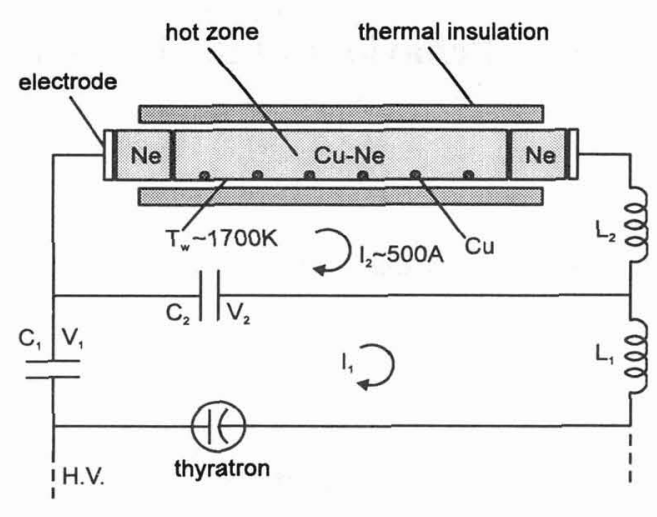

Figure 1: Schematic diagram of the copper laser and external pulse forming network.

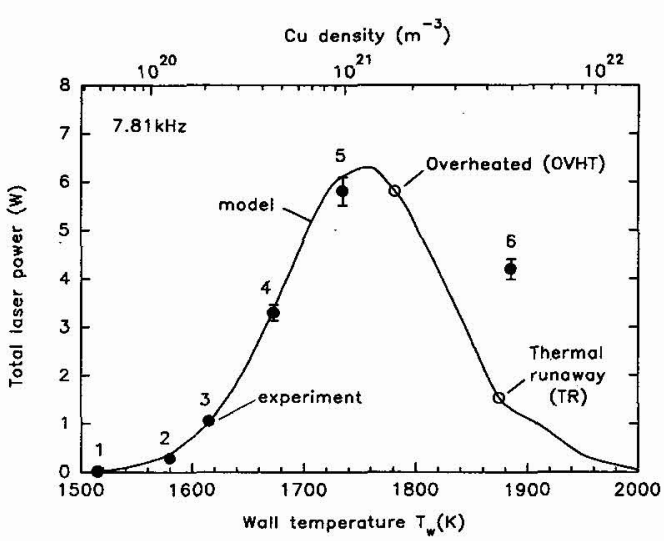

Figure 2: Calculated laser output power (solid line) as a function of wall temperature for a fixed $\mathrm{prf}=7.81 \mathrm{kHz}$ compared with experimental data [14] (filled circles). Specific operating conditions are represented by the legend (1)-(6), OVHT (overheated) and TR (thermal runaway).

neutrals as a function of time and radial position assuming axial homogeneity and radial symmetry for the $\mathrm{Cu}-\mathrm{Ne}$ "hot zone" (fig 1). The behaviour of the pure neon plasma in the two "cold-zone" regions extending beyond the thermal insulation layers is modelled in a similar manner. The plasma skin effect relating to the temporal evolution of the electric field across the tube radius is taken into account for the $\mathrm{Cu}-\mathrm{Ne}$ zone. The temperature of the plasma tube wall $\mathrm{T}_{\mathrm{w}}$ (controlling the copper density) is evaluated from the calculated total power deposited in the discharge plasma. The model has also been adapted to include a kinetics scheme for an additional 10 hydrogen species $\left(\mathrm{H}_{2}, \mathrm{H}, \mathrm{H}, \mathrm{H}_{2}(\mathrm{v}), \mathrm{H}^{*}, \mathrm{H}^{+}\right)$to investigate the known performance enhancement of CVLs using $\mathrm{H}_{2}$ admixtures [12]. Results from the model have been compared with experimental data from a small bore CVL (see table I), including spatially and temporally resolved "hook" population measurements. The calculations have been carried out using a CRAY Y-MP/EL and Microvax 3100 using a standard IMSL package [13] to solve the set of first-order coupled differential equations based on the backward differentiation or GEAR method for "stiff" equations. Self-consistent results for around 350 different operating conditions have been generated requiring 2500 CPU hours use on the CRAY.

\begin{tabular}{ll}
\hline Table I & \\
\hline Cu-Ne hot-zone length & $75 \mathrm{~cm}$ \\
Neon cold-zone length (total) & $18 \mathrm{~cm}$ \\
Plasma tube diameter & $1.8 \mathrm{~cm}$ \\
Neon pressure & $40 \mathrm{mb}$ \\
Storage/Peak capacitance $\mathrm{C}_{1} / \mathrm{C}_{2}$ & $6 / 2 \mathrm{nF}$ \\
Circuit Inductances $\mathrm{L}_{1} / \mathrm{L}_{2}$ & $1.0 / 0.5 \mu \mathrm{H}$ \\
Mirror reflectivities & $0.04 / 1.0$ \\
Optical cavity length & $175 \mathrm{~cm}$ \\
\hline
\end{tabular}

\section{RESULTS AND DISCUSSION}

The total laser output power $(510.6 \mathrm{~nm}+578.2 \mathrm{~nm})$ calculated by the model as a function of the wall temperature $T_{w}$ for a fixed pulse repetition frequency (prf) is shown in fig 2 . In the model as in actual experiments, changes in $T_{w}$ occur as a result of varying the storage capacitor voltage $V_{1}$ (and thus the 
deposited power) whilst keeping the capacitance $\mathrm{C}_{1}$ and prf constant. Experimental points are plotted for six distinct operating conditions, and are in close agreement with the model data for $T_{w}<1750 \mathrm{~K}$. In the region where the output power is falling with increasing wall temperature $\left(T_{w}>1750 \mathrm{~K}\right)$, the plasma tube was observed (experimentally) to exhibit the well known thermal runaway behaviour[14]. Under these conditions, the laser output slowly diminishes (turning yellow) over a period of a few minutes as the wall temperature steadily climbs by tens of degrees, despite the fact that the charging voltage $V_{1}$ is kept constant. Throughout the remainder of this paper, two distinct operating points are used for this unstable regime as depicted in fig. 2 ; to represent a slightly overheated (OVHT) wall temperature $\left(\mathrm{T}_{\mathrm{w}}=1781 \mathrm{~K}\right)$, and conditions of thermal runaway (TR) $\left(\mathrm{T}_{\mathrm{w}}=1875 \mathrm{~K}\right)$. The temporal evolution of the axial $\mathrm{Cu}^{2} \mathrm{P}_{3 / 2}$ population density, the upper level for the 510.6 laser transition, is shown in fig. 3 and compared with experimental hook measurements for specific operating conditions in fig.1. Once again the agreement is good for the data sets denoting different operating conditions and wall temperatures, notably for the experimental data and the model results for the.OVHT condition with $T_{w}=1781 \mathrm{~K}$ (fig. 2 ).

Insight into the cause of the observed thermal runaway behaviour of the plasma tube can be gained by calculating the fractional energy deposition in the $\mathrm{Cu}-\mathrm{Ne}$ zone, based on the initial energy in the storage capacitor $\left(1 / 2 \mathrm{C}_{1} \mathrm{~V}_{1}^{2}\right)$, as shown in fig.4. For wall temperatures corresponding to the leading edge of the output power curve in fig. $1\left(\mathrm{~T}_{w}<1740 \mathrm{~K}\right)$, this fraction decreases slightly with increasing $\mathrm{T}_{w}$ due to an overall fall in the plasma resistance (increased plasma conductivity) as the copper density rises. For a given point on the curve in this region characterised by a fixed input power (or fixed $\mathrm{V}_{1}$ ), any small perturbation of the copper density in the $\mathrm{Cu}-\mathrm{Ne}$ zone (caused by a hot-spot on the wall for example) will produce a change in the fractional power deposition. The ensuing change in the wall temperature will occur in the opposite sense, tending to restore the copper density to the original value, thus ensuring thermally stable operation through negative feedback. For higher temperatures beyond the local minimum of the curve $\left(T_{w}>1740 K\right)$, the fractional power deposition increases with rising $T_{w}$, due to an overall increase of the plasma resistance or lowering of plasma conductivity. Any small perturbation of the copper density (at fixed $V_{c}$ ) will result in an increase in the fractional power deposition and a rise in $T_{w}$, leading to a further increase of the copper vapour density establishing a positive feedback mechanism. Under such conditions, the plasma tube will eventually exhibit the well-known thermal runaway behaviour. Experimentally, it is known that in the unstable regime before thermal runaway occurs, the wall temperature becomes very sensitive to small changes in the charging voltage $V_{1}$. In the model for calculations in the temperature range $T_{w}>1800 \mathrm{~K}$, the wall temperature calculated from the deposited power is also extremely sensitive to small increases in $V_{1}$ beyond $9.2 \mathrm{kV}$ as shown in fig.4. Indeed

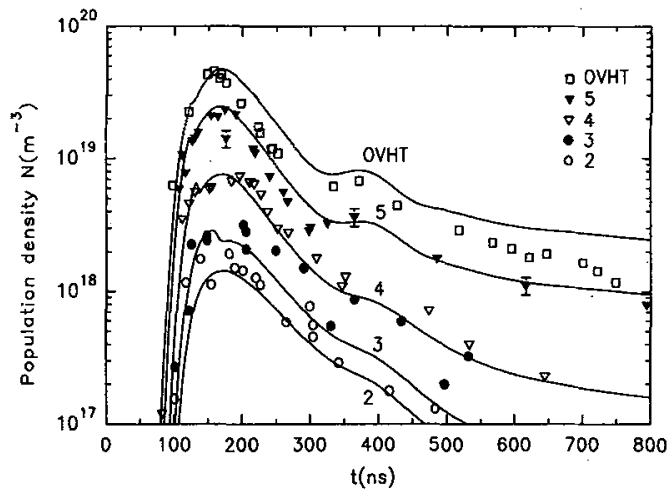

Figure 3. Calculated temporal evolution of the axial population density of the ${ }^{2} \mathrm{P}_{3 / 2}$ upper laser level during the excitation phase for different operating conditions at $\mathrm{prf}=7.81 \mathrm{kHz}$ compared with hook measurements [14]. The legend (2)-(5) and OVHT is the same as in fig. 2 .

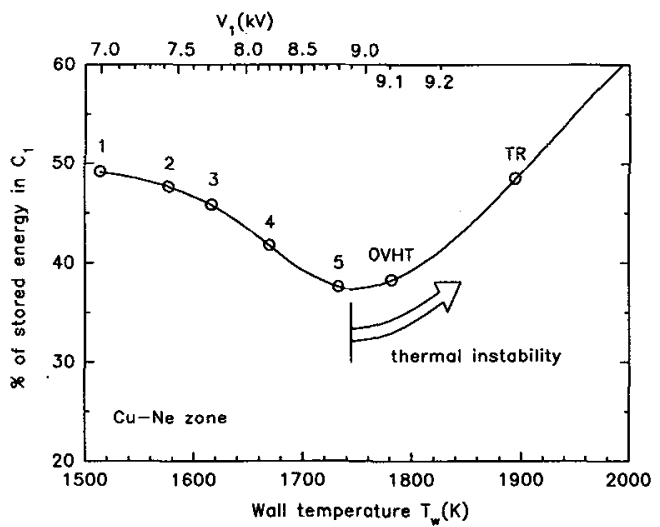

Figure 4. Calculated fractional power deposition in the $\mathrm{Cu}$ Ne hot zone as a function of the wall temperature at prf= $7.81 \mathrm{kHz}$. The legend (1)-(5), OVHT and TR is the same as 
for $T_{w}>1850 \mathrm{~K}$, the self-consistent results asymptotically converge to a small but consistent pulse-to-pulse increase of the wall temperature $\left(\Delta \mathrm{T}_{w} \sim 0.1 \mathrm{~K}-1.0 \mathrm{~K}\right)$ at fixed $\mathrm{V}_{1}$. It was not possible to follow the plasma evolution over a large number of cycles $(>100)$ (to simulate thermal runaway) due to limitations in computing power.

The fractional power deposition in the $\mathrm{Cu}-\mathrm{Ne}$ zone is directly related to the plasma resistance or the equivalent plasma conductivity $\sigma$ :

$$
\sigma=\frac{N_{e} e^{2}}{3 k T_{e}}\left\langle\frac{v_{e}}{\Sigma_{j} N_{j} \sigma_{m j}}\right\rangle
$$

where $\sigma_{m j}$ is the electron momentum transfer cross-section with the species $N_{j}, v_{c}$ is the electron velocity, and $\mathrm{k}$ is the Boltzmann constant. At low $\mathrm{T}_{w}(<1740 \mathrm{~K})$ corresponding to the regime of thermal stability, the conductivity decreases with rising $T_{w}$ because there is a gradual drop in the peak electron temperature $T_{e}$ and a rise in the pre-pulse electron density. The drop in the peak $T_{c}$ occurs as a result of the increasing importance of inelastic collisions with copper atoms which have relatively large impact cross-sections compared with the neon buffer gas. At maximum output power (at $T_{w}=1760 \mathrm{~K}$ ), the peak axial $\mathrm{T}_{e}$ is around $3.3 \mathrm{eV}$, in agreement with semi-empirical calculations reported elsewhere (peak $\mathrm{T}_{c} \sim 2.9 \mathrm{eV}$ ) for a CVL with a similar size bore[15]. At high $\mathrm{T}_{w}(>1740 \mathrm{~K})$ in the regime of thermal instability, the plasma conductivity begins to fall again due mainly to the increasing importance of e-Cu momentum transfer collisions, and partly to a drop in the pre-pulse electron density. The fall in output power at high $\mathbf{T}_{\mathrm{w}}$ $(>1800 \mathrm{~K})$ is due to a fall in the electron temperature rather than a build-up of population in the metastable lower levels since the density ratios with respect to the ground state (eg. ${ }^{2} \mathrm{D}_{5 / 2}{ }^{2} \mathrm{~S}_{1 / 2}$ ) decrease with increasing $T_{w}$. As a further illustration of the influence of the copper atoms on the plasma conductivity, the ratio $\sigma / \mathrm{N}_{\mathrm{e}}$ (on axis) is plotted in fig. 5 for the seven specific operating conditions defined in fig. 2 . At low $\mathrm{T}_{\mathrm{w}}$ corresponding to conditions (1)-(4), the curves are practically identical because the momentum transfer collision frequency is dominated by collisions with neon atoms. However, the peak electron temperatures are lower due to e-Cu inelastic collisions as more copper is introduced into the plasma (higher $T_{w}$ ), so the $\sigma / N_{c}$ values are higher on average. For condition (5) close to maximum output power, $\sigma / N_{c}$ values are slightly lower, particularly at low $T_{c}$. This follows from the e-Cu momentum transfer cross-section which is peaked around $\mathrm{E}=0.5 \mathrm{eV}$ and thereafter falls rapidly (fig 5 , inset) whereas the same

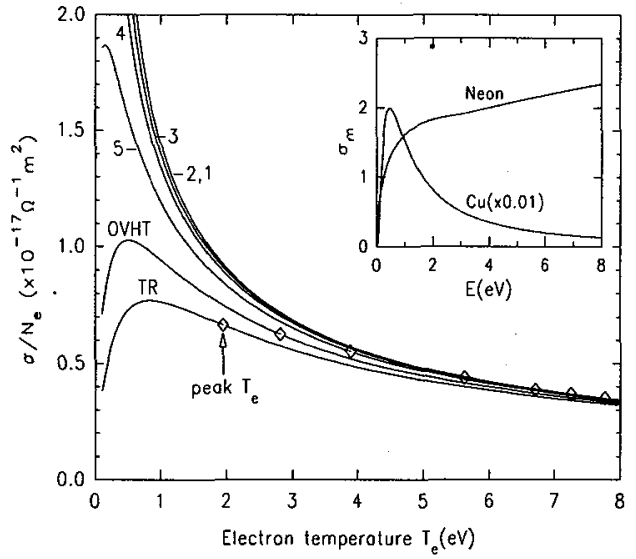

Figure 5. Calculated plasma conductivity parameter $\sigma / \mathrm{N}_{\mathrm{c}}$ as a function of the electron temperature $T_{c}$ for different operating conditions (1)-(5), OVHT and TR ( $p r f=7.81 \mathrm{kHz}$ ). The energy dependence of the momentum transfer cross-section for e-Ne and e-Cu collisions is shown inset (units are $10^{-20} \mathrm{~m}^{2}$ ). 


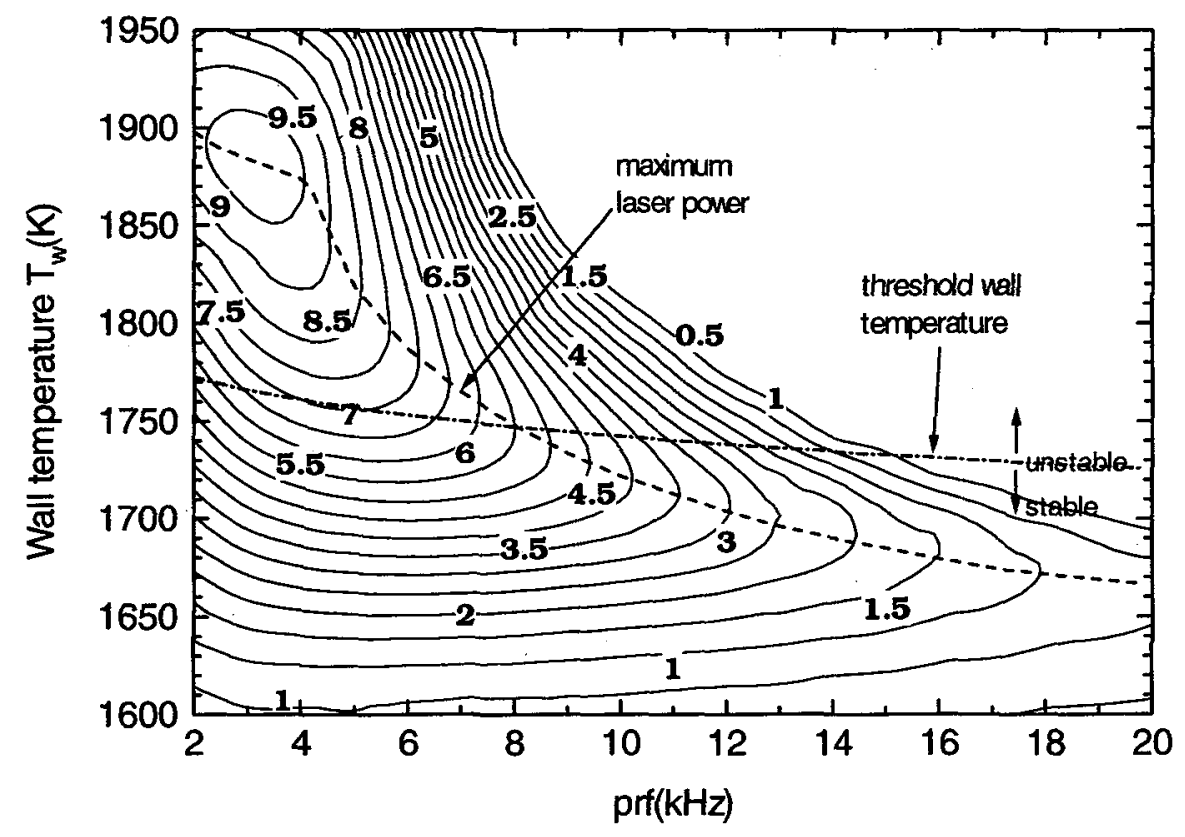

Figure 6. Calculated total laser power (shown in watts on each contour) as a function of prf and wall temperature (buffer gas pressure is $40 \mathrm{mb}, C_{1} / C_{2}=6 / 2 n F$ ).

cross-section for neon climbs more gradually to reach a peak around $\mathrm{E}=30 \mathrm{eV}$. For conditions (OVHT) and (TR) in the unstable regime $\left(\mathrm{T}_{\mathrm{w}}>1740 \mathrm{~K}\right), \sigma / \mathrm{N}_{\mathrm{c}}$ values are strongly dependent on the copper density at low $T_{c}(<2 e V)$, although less so at higher $T_{c}$. Thus, the e-Cu momentum transfer collisions cause a reduction in the overall plasma conductivity giving rise to a larger fractional power deposition (fig.4), with the reduction in the peak $T_{\mathrm{e}}$ acting as a compounding factor. To delay the onset of thermal instability towards higher wall temperatures and larger copper densities, it is therefore necessary to increase the density of neon atoms and/or increase the peak $T_{e}$ to diminish the influence of the e-Cu momentum transfer collisions in determining the $\sigma / \mathrm{N}_{\mathrm{e}}$ values.

A detailed parametric study of the CVL performance has also been carried out with the total output power calculated as a function of both $T_{w}$ and prf as shown in fig.6. The results indicate that the maximum attainable output power should be around $10 \mathrm{~W}$ in the prf range $2-4 \mathrm{kHz}$, diminishing slowly as the prf increases. Furthermore, the optimum wall temperature for maximum output power is lower at higher prfs, consistent with the smaller excitation pulse energies at higher prfs. A "threshold" wall temperature can be defined as that corresponding to the lowest point of the curve in fig. 4 marking the transition between thermally stable and unstable modes of operation. As shown in fig.6, this threshold temperature exhibits a relatively weak dependence on the prf over the range $2-20 \mathrm{kHz}$, in the range $1725 \mathrm{~K}$ $1775 \mathrm{~K}$. Consequently, the copper density at the tube wall will be limited to a similarly narrow range $\left(9 \times 10^{20}-1.6 \times 10^{21} \mathrm{~m}^{-3}\right)$. These results suggest that CVL operation for wall temperatures above the threshold, would not be possible in reality owing to thermal instability of the plasma tube, leading to thermal runaway.

The calculated maximum output powers for CVL operation within the stable regime are shown in fig.7a together with those at low prf $(2-8 \mathrm{kHz})$ that fall in within the unstable regime. Experimentally, laser output powers are found to optimise around $6 \mathrm{~W}$ for prfs $5-8 \mathrm{kHz}$ (fig.7a), in agreement with the calculated values for operation within the stable regime. These results are consistent with the hypothesis that the 


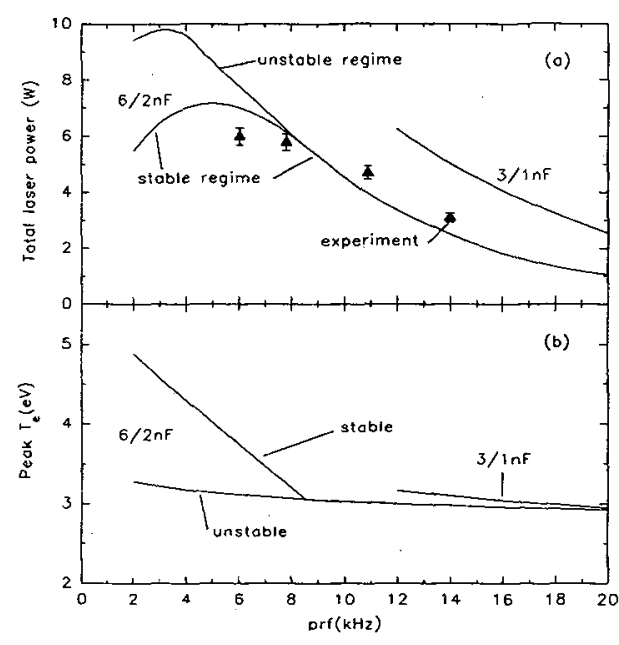

Figure 7. (a) Calculated maximum laser output power as a function of prf for operation in the stable and unstable regimes compared with experimental data [11] (triangles), (b) calculated peak electron temperature as a function of prf.

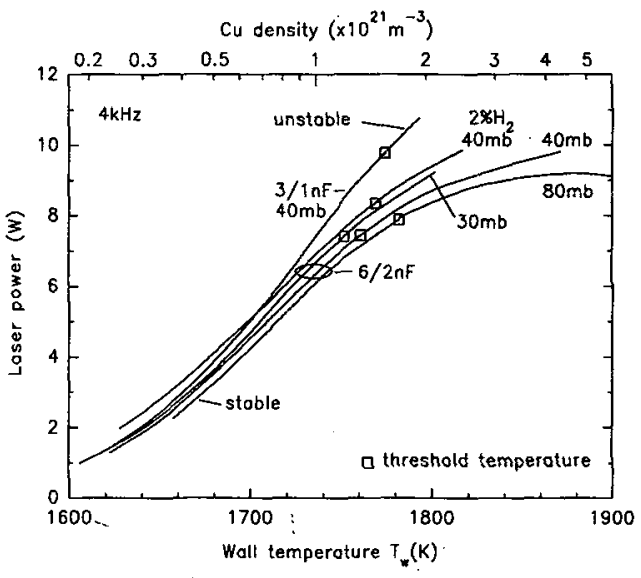

Figure 8. Calculated total laser power as a function of wall temperature at $4 \mathrm{kHz}$ for different neon pressures, capacitance values, and with a $2 \% \mathrm{H}_{2}$ admixture.

laser output powers for $\mathrm{prf}<8 \mathrm{kHz}$ are indeed limited by thermal instability which sets an operating limit on the wall temperature and copper density.

Another important result is that the calculated peak electron temperature, at the radial position giving maximum laser pulse energy, is almost constant $\left(\mathrm{T}_{\mathrm{c}}=2.9-3.1 \mathrm{eV}\right)$ at high prf $(>8 \mathrm{kHz})$ as shown in fig. $7 \mathrm{~b}$. For prf $<8 \mathrm{kHz}$, the peak $T_{c}$ rises linearly to $5 \mathrm{eV}$ with decreasing prf when in the stable regime, but would remain around $3 \mathrm{eV}$ if it were possible to operate in the unstable regime at the maximum power level. The model results suggest that fundamentally, there is an optimum peak electron temperature around $3 \mathrm{eV}$ for maximising the laser output power. In the absence of thermal instability effects, maximum output power is achieved by matching the copper density to the available excitation pulse energy to maintain the peak $T_{c}$ at $\sim 3 \mathrm{eV}$. Additional calculations have also been performed using smaller values of circuit capacitance $(3 / 1 \mathrm{nF})$ as usually employed experimentally to enhance laser output powers at high prfs (fig 7). Although the output powers at high prf are nearly doubled, the peak $T_{c}$ remains at the optimum around $3 \mathrm{eV}$.

Changes in the predicted laser output power for slightly different operating conditions than were standard ( $40 \mathrm{mb}, \mathrm{C}_{1} / \mathrm{C}_{2}=6 / 2 \mathrm{nF}$ ) have been calculated for a prf of $4 \mathrm{kHz}$ (fig. 8 ) to investigate the influence on the threshold wall temperature which limits the attainable copper density at this prf. There appears to be little difference in the output power for gas pressures in the range $30-80 \mathrm{mb}$, although operation at $80 \mathrm{mb}$ enables the threshold wall temperature to rise by $20 \mathrm{~K}$ (a $20 \%$ increase in copper density) before instability sets in. It is interesting to note that the overall laser output power is slightly higher at $80 \mathrm{mb}$ than at $40 \mathrm{mb}(+0.4 \mathrm{~W})$ because the small drop in output power at fixed $\mathrm{T}_{w}$ is more than offset by the increased threshold wall temperature. By using smaller capacitance values $\left(C_{1} / C_{2}=3 / 1 n F\right)$ and higher charging voltages $\left(\mathrm{V}_{1}\right)$, the output power at fixed $\mathrm{T}_{\mathrm{w}}$ is significantly increased, and the threshold temperature also rises by $13 \mathrm{~K}$ (a $12 \%$ increase in copper density). Such changes are consistent with an increase in the peak $T_{c}$ resulting from the higher charge voltages $V_{1}$ (and subsequently the applied tube voltage $\mathrm{V}_{2}$ ). The addition of a $2 \%$ admixture of hydrogen to the buffer gas also increases the laser output power and threshold wall temperature $(+7 \mathrm{~K})$ at $4 \mathrm{kHz}$ compared with the standard case. In this case, the hydrogen improves the interpulse plasma relaxation rate giving rise to a higher tube voltage $V_{2}$ (and peak $\mathrm{T}_{\mathrm{c}}$ ). 


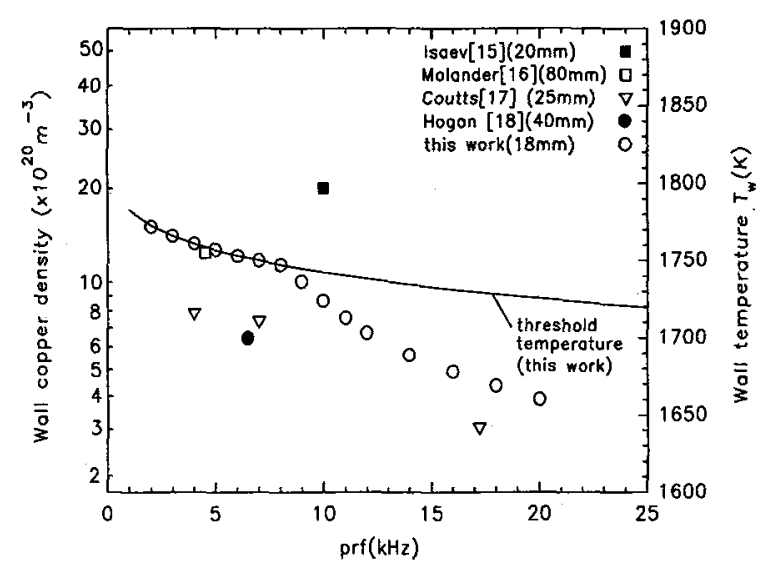

Figure 9. Copper density at the plasma tube wall as a function of the prf for different copper lasers.

As discussed above, the results shown in fig 6 suggest that the wall temperature at the threshold of thermal instability (and associated copper density) falls within the narrow range $T_{w}=1725 \mathrm{~K}-1775 \mathrm{~K}\left(\mathrm{Cu}_{\text {wall }}\right.$ $\left.=9 \times 10^{20}-1.6 \times 10^{21} \mathrm{~m}^{-3}\right)$ for the prfs investigated $(2-20 \mathrm{kHz})$. It is interesting to note that direct measurements of the copper density at the wall in elemental CVLs from different laboratories [15-18] do not exceed these limits, in the majority of cases, as shown in fig.9. This is despite the fact that the devices differ greatly in the bore size, in their electrical power loading and in the design of the thermal insulation. (In reality, each CVL device will have its own characteristic range of threshold wall temperatures marking stable/unstable operation depending on the specific operating parameters of the laser). One exception is the CVL of Isaev[15] where a relatively high copper density $\left(\mathrm{Cu}_{\text {wall }}=20 \times 10^{20} \mathrm{~m}^{-3}\right)$ was reported. The stable operation of the laser at this high copper density is undoubtedly related to the very high neon gas pressure employed $(300 \mathrm{mb})$ in their device which would delay the onset of thermal runaway until higher wall temperatures are reached (see fig 8 ).

\section{SUMMARY}

A self-consistent model has been used to map the parametric behaviour of an elemental copper vapour laser for optimum and non-optimum operating conditions. The results show that the two most important plasma parameters affecting laser performance are the ground state copper density, and the peak electron temperature. Fundamentally, there appears to be an optimum peak electron temperature of $\sim 3 \mathrm{eV}$ at any given point in the plasma for maximising the laser power. The results also confirm the existence of a threshold wall temperature (and copper density) above which the plasma tube exhibits a thermal runaway behaviour due to a positive feedback mechanism between the deposited electrical power, the wall temperature and the copper density. This phenomenon occurs when the copper atom density is high enough to directly influence the plasma conductivity through e-Cu momentum transfer collisions. The threshold wall temperature falls within a narrow range $1725 \mathrm{~K}<\mathrm{T}_{\mathrm{w}}<1775 \mathrm{~K}$ for the prf range $2-20 \mathrm{kHz}$, corresponding to a copper density of $9 \times 10^{20}-1.6 \times 10^{21} \mathrm{~m}^{-3}$. At low prf values $(<8 \mathrm{kHz})$, thermal runaway limits the attainable copper density (and laser output power) to values below optimum with respect to the peak electron temperature. It is shown that the threshold wall temperature can be increased allowing operation at elevated copper densities (leading to increased laser output power) by increasing the neon buffer gas density and/or by increasing the peak $T_{c}$ through the use of hydrogen admixtures and low circuit capacitances/high charge voltages. 


\section{Acknowledgments}

The author thanks D.J.W. Brown (Macquarie University) for generously providing unpublished experimental data and K.F. Scheibner (Lawrence Livermore National Laboratory) for providing unpublished cross-sections for e-Cu inelastic collisions. I am also indebted to Prof. M.A. Morrison (University of Okalahoma) for providing a detailed set of cross-sections for electronic excitation/deexcitation of the ro-vibrational states of $\mathrm{H}_{2}$. This work was supported by the Australian Research Council in the form of an Australian Research Fellowship (1994-1998).

\section{References}

[1] Coutts D.W. and Brown D.J.W. IEEE J.Sel.Topics Quantum Electron., 3, (1995).

[2] Knowles M.H. and Webb C.E. Opt. Lett. 18 (1993) 607.

[3] Kapitan D., Booth H., Murray G.J., Hogan G.P. and Webb C.E. Proc. SPIE 3092 (1997) 732.

[4] Glover A.C.J., Illy E.K. and Piper J.A. IEEE J.Sel.Topics Quantum Electron. 1 (1995) 830.

[5] Kupfer R., Bergmann H. and Lingenauer M. Proc. SPIE 1598 (1991) 46.

[6] Kushner M.J. and Warner B.E. J.Appl.Phys. 54 (1983) 2970.

[7] Borovich B.L. and Yurnchenko N.I., Sov. J.Quantum Electron. 14 (1985) 1391.

[8] Kushner M.J., IEEE J. Quantum Electron. QE-17 (1981) 1555.

[9] Carman R.J., Brown D.J.W and Piper J.A. IEEE J. Quantum Electron. QE-30 (1993) 1876.

[10] Brown D.J.W., Kunnemeyer R. and McIntosh A.I. IEEE J. Quantum Electron. QE-26 (1990) 1609.

[11] Brown. D.J.W. (unpublished data).

[12] Carman R.J. (to be published)

[13] IMSL Library of Fortran subroutines, "DGEAR differential equation solver" IMSL Inc. (1985).

[14] Brown D.J.W., PhD dissertation, University of New England, Armidale, Australia, (1988).

[15] Isaev A.A. and Petrash G.G. Proc. SPIE 2110 (1993) 2.

[16] Molander W.A. Proc. SPIE 1041 (1989) 11.

[17] Copper ground state densities have been inferred from comparisons of the performance of an existing laser device and modelled results for that device. See Coutts D.W., PhD dissertation, Macquarie University, Australia (1993).

[18] Hogan G.P. PhD Dissertation, University of Oxford, England, (1994). 